\title{
Lethal dysregulation of energy metabolism during embryonic vitamin $\mathrm{E}$ deficiency
}

\author{
Melissa McDougall $^{\mathrm{a}, \mathrm{b}}$, Jaewoo Choi ${ }^{\mathrm{a}}$, Hye-Kyeong Kim ${ }^{\mathrm{c}}$, Gerd Bobe ${ }^{\mathrm{a}}$, J. Frederik Stevens ${ }^{\mathrm{a}, \mathrm{d}, \mathrm{e}}$, \\ Enrique Cadenas ${ }^{\mathrm{f}}$, Robert Tanguay ${ }^{\mathrm{e}, \mathrm{g}, \mathrm{h}}$, Maret G. Traber ${ }^{\mathrm{a}, \mathrm{b}, \mathrm{e}, *}$ \\ ${ }^{a}$ Linus Pauling Institute, Oregon State University, Corvallis, OR 97331, USA \\ b College of Public Health and Human Sciences, Oregon State University, Corvallis, OR 97331, USA \\ c The Catholic University of Korea, Seoul, Republic of Korea \\ d College of Pharmacy, Oregon State University, Corvallis, OR 97331, USA \\ e Environmental Health Sciences Center, Oregon State University, Corvallis, OR 97331, USA \\ ${ }^{\mathrm{f}}$ University of Southern California, School of Pharmacy, Los Angeles, CA 90089, USA \\ ${ }^{g}$ Environmental and Molecular Toxicology, Oregon State University, Corvallis, OR 97331, USA \\ h Sinnhuber Aquatic Research Laboratory, Oregon State University, Corvallis, OR 97331, USA
}

\section{A R T I C L E I N F O}

\section{Keywords:}

$\alpha$-tocopherol

Docosahexaenoic acid

Methyl donors

Mitochondria

Oxygen consumption

Phosphatidylcholine

\begin{abstract}
A B S T R A C T
Vitamin E ( $\alpha$-tocopherol, VitE) was discovered in 1922 for its role in preventing embryonic mortality. We investigated the underlying mechanisms causing lethality using targeted metabolomics analyses of zebrafish VitE-deficient embryos over five days of development, which coincided with their increased morbidity and mortality. VitE deficiency resulted in peroxidation of docosahexaenoic acid (DHA), depleting DHA-containing phospholipids, especially phosphatidylcholine, which also caused choline depletion. This increased lipid peroxidation also increased NADPH oxidation, which depleted glucose by shunting it to the pentose phosphate pathway. VitE deficiency was associated with mitochondrial dysfunction with concomitant impairment of energy homeostasis. The observed morbidity and mortality outcomes could be attenuated, but not fully reversed, by glucose injection into VitE-deficient embryos at developmental day one. Thus, embryonic VitE deficiency in vertebrates leads to a metabolic reprogramming that adversely affects methyl donor status and cellular energy homeostasis with lethal outcomes.
\end{abstract}

\section{Introduction}

Vitamin E ( $\alpha$-tocopherol, VitE) was discovered in 1922 because it prevented embryonic mortality in rats [1], but the involved mechanisms remain unknown. VitE is a lipophilic antioxidant [2], protecting long-chain polyunsaturated fatty acids, especially docosahexaenoic acid (DHA) from lipid peroxidation [3,4]. Is VitE's essentiality due to its antioxidant function? A leading theory argues VitE is an integral part of an "antioxidant network" [5], where the one-electron VitE oxidation product (generated by its scavenging peroxyl radicals) is reduced by ascorbic acid. Reduction of the ascorbyl radical utilizes other antioxidants, especially glutathione (GSH). The subsequent reduction of GSSG (the GSH oxidization product) uses endogenous reducing equivalents, primarily NADPH, to serve as the final electron-acceptor [5]. This close association between GSH and VitE is further emphasized by studies of phospholipid hydroperoxide glutathione peroxidase (GPx4), a selenium-dependent peroxidase that reduces phospholipid hydroperoxides [6]. Could dysregulation of this antioxidant network cause embryonic mortality? Maternal VitE supplementation in mice prevents GPx4 knockdown-induced lethality in offspring [7], suggesting that VitE is necessary both 1) to generate lipid hydroperoxides from peroxyl radicals (the lipid hydroperoxides then function as GPx4 substrates) and 2) in the absence of GPx4 to prevent alkoxyl radicals, which arise from the spontaneous oxidation of lipid hydroperoxides, from reinitiating lipid peroxidation.

In humans, VitE deficiency increases early miscarriage risk [8], which poses public health concerns since estimates of inadequate dietary VitE intakes exceed $80 \%$ of the global $\geq 14$ year-old population

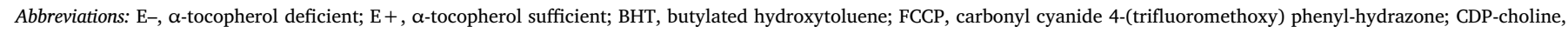

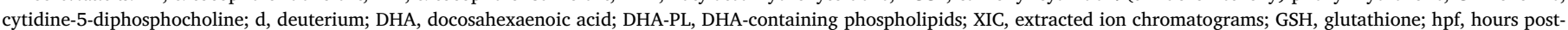

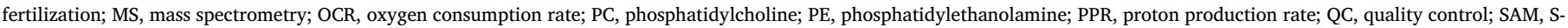
adenosylmethionine; TOF, time-of-flight; TCA, tricarboxylic acid; VitE, vitamin E; ZAAP, zebrafish acquisition and analysis program

* Corresponding author at: Linus Pauling Institute, Oregon State University, Corvallis, OR 97331, USA.

E-mail address: maret.traber@oregonstate.edu (M.G. Traber). 
[9]. Using our VitE-deficient zebrafish model as a tool, we undertook solving the mystery of why VitE is a necessary nutrient, especially during vertebrate embryonic development.

\section{Materials and methods}

\subsection{Study design}

All experiments were performed in duplicate. All data trends and specific outcomes contained herein matched between the first and second experimental replicates. In order to maximize consistency, the results we report are all from the second set of experiments. Additional supplementary data and the complete data set is available online.

\subsection{Materials and reagents}

Reagents used for metabolomics analyses included: methanol and ultra-pure water (LC-MS grade, EMD Millipore, Gibbstown, NJ); formic acid, acetic acid (Optima LC/MS grade; Fisher Chemical, Pittsburgh, PA); and butylated hydroxytoluene (BHT, TCI America; Portland, OR), as well as zirconium oxide beads (Next Advance; Averill Park, NY). Deuterium (d) labeled internal standards DHA- $\mathrm{d}_{5}$, ARA- $\mathrm{d}_{8}$, EPA- $\mathrm{d}_{5}$, LA$\mathrm{d}_{4}$, and $9(S)$-HODE- $\mathrm{d}_{4}$ (Cayman Chemical, Ann Arbor, MI) were used for quantification of total and free fatty acids, and oxidized DHA derivatives, respectively. Sterile $d_{6}$ - $\alpha$-tocopherol emulsion (Fresenius-Kabi, Graz, Austria) and D- $(+)$-glucose (Sigma Aldrich, St. Louis MO) were used for embryo microinjection rescue studies. Reagents used for bioenergetics profiling included: oligomycin (Cayman Chemicals; Ann Arbor, MI), carbonyl cyanide 4-(trifluoromethoxy) phenyl-hydrazone (FCCP), and sodium azide (Sigma-Aldrich; St. Louis, MO).

\subsection{Zebrafish husbandry and diets}

The Institutional Animal Care and Use Committee of Oregon State University approved this protocol (ACUP Number: 4344). Tropical 5D strain zebrafish were housed in the Sinnhuber Aquatic Research Laboratory. Adults were kept at standard laboratory conditions of $28{ }^{\circ} \mathrm{C}$ on a 14-h light/10-h dark photoperiod in fish water consisting of reverse osmosis water supplemented with a commercially available salt (Instant Ocean $^{\circledast}$ ) to create a salinity of 600 microsiemens [11], adjusted to $\mathrm{pH}$ 7.4. At 55 days post-fertilization (dpf), adult zebrafish were randomly allocated to one of two diet groups, $\alpha$-tocopherol deficient (E-) or $\alpha$-tocopherol sufficient $(\mathrm{E}+$ ), for the duration of the study [12]. The defined diets, which contained only fatty acids with 18 or fewer carbons and no more than 3 double bonds, were prepared with the vitamin C source as StayC $(500 \mathrm{mg} / \mathrm{kg}$, Argent Chemical Laboratories Inc., Redmond, WA) and without (E-) or with added $\alpha$ tocopherol (E+, $500 \mathrm{mg} R R R$ - $\alpha$-tocopheryl acetate $/ \mathrm{kg}$ diet, ADM, Decatur, IL), as described previously [12,13]. Diets were stored at $-20{ }^{\circ} \mathrm{C}$ until fed to the adult zebrafish.

$\mathrm{E}-$ and $\mathrm{E}+$ embryos were obtained from adult fish fed either the Eor $\mathrm{E}+$ diet, respectively, for a minimum of 80 days up to 9 months. Embryos were obtained through natural group spawning, collected, staged [11], and kept in standard embryo media (EM; as described [14]). Embryos used for biochemical analysis, described below, were euthanized prior to sampling by cold exposure (placed on ice for a minimum of $30 \mathrm{~min}$ ). Note that embryos are not fed. For all experiments, the $\mathrm{E}+$ embryos are considered the control condition; lab embryos also were used as an additional control to monitor embryo quality (data not shown).

\subsection{Vitamin $E$ and ascorbic acid analyses}

Using high-pressure liquid chromatography with electrochemical detection, diet and embryo $\alpha$-tocopherol [15] and ascorbic acid [16] were determined. Measured $\alpha$-tocopherol concentrations in the $\mathrm{E}$ - and
$\mathrm{E}+$ diets were $0.45 \pm 0.01$ and $369 \pm 2 \mathrm{mg} / \mathrm{kg}$, respectively; vitamin C was $143 \pm 16 \mathrm{mg}$ ascorbic acid $/ \mathrm{kg}(\mathrm{n}=3$ replicate samples measured for each diet). This level of dietary vitamin $\mathrm{C}$ has been found to be adequate for the zebrafish [17].

\subsection{Evaluation of phenotypic and developmental progress}

At $24 \mathrm{~h}$ post-fertilization (hpf), embryos were assessed for viability, developmental progression and spontaneous movements (earliest behavior in zebrafish), using the zebrafish acquisition and analysis program (ZAAP). ZAAP is a custom program designed to inventory, acquire, and manage zebrafish data, and was used to collect 18 developmental endpoints, as either present or absent (i.e. binary responses were recorded, described below [18]).

Developmental progression is considered perturbed if zebrafish are delayed more than $12 \mathrm{~h}$ compared to control animals. Spontaneous movements are assessed over a $2 \mathrm{~min}$ period and are considered perturbed if there is a lack of embryonic contractions and/or movement. At 96 and 120 hpf, larval morphology (body axis, eye, snout, jaw, otic vesicle, notochord, heart, brain, somite, fin, yolk-sac, trunk, circulation, pigment, and swim bladder) was evaluated and recorded and behavioral endpoints (motility, tactile response) were thoroughly evaluated. If the embryo was dead at either 24 or by $96-120 \mathrm{hpf}$, the non-mortality endpoints were not included in the evaluations. All images were taken using a Keyence BZ-700X microscope with a $2 \times$ objective lens under standard bright-field conditions.

\subsection{Behavioral assessments}

Locomotor activity $[18,19]$ was measured in a total of $n=128$ embryos per VitE group using Viewpoint Zebrabox (software version 3.0, Viewpoint Life Sciences, Lyon, France). At $96 \mathrm{hpf}$, the plates containing the embryos were placed in a Viewpoint ZebraBox and embryo locomotor activity was assessed using the "tracking" setting during alternating periods of light and dark, a modification of [20]. Embryos subjected to this test typically move less during the light periods and more during dark periods, and behavioral differences can be determined by comparing distances moved during the light and/or dark periods. Locomotor activity in response to the light/dark transition was tracked during $3 \mathrm{~min}$ periods of alternating light and dark for a total of $24 \mathrm{~min}$. The integration time was set to $6 \mathrm{~s}$ to increase statistical power. A high definition camera (30 frames/second) tracked the total movement (swim distance, millimeters) in response to the multiple light-dark transitions.

\subsection{Extraction and LC-MS/MS for metabolomic analysis}

At $12 \mathrm{hpf}$, E- and E + embryos were transferred one embryo per well into 96 well plates containing $100 \mu \mathrm{L}$ EM per well. Following 24, 48, 72, and $120 \mathrm{hpf}$, embryos $(n=15$ per replicate, $n=4$ replicates per group) were transferred to $1.5 \mathrm{~mL}$ Eppendorf tubes, covered with EM, and kept on ice for $30 \mathrm{~min}$ to euthanize the animals. EM was carefully removed to prevent loss of embryos and samples were stored at $-80{ }^{\circ} \mathrm{C}$ overnight. To extract embryos for metabolomics analyses, solvent (300 $\mu \mathrm{L} 80: 20 \mathrm{v} / \mathrm{v}$ methanol:water) was added, then sample extracts were homogenized with $0.5 \mathrm{~mm}$ zirconium oxide beads using a countertop bullet blender for $6 \mathrm{~min}$. Following $15 \mathrm{~min}$ incubation on ice, the extracts were centrifuged at $4{ }^{\circ} \mathrm{C}$ at $15,000 \times g$ for $13 \mathrm{~min}$. Aliquots $(200 \mu \mathrm{L})$ of the upper layer were transferred individually to new tubes and stored at $-80{ }^{\circ} \mathrm{C}$ until analysis via LC-MS/MS. To ensure the stability and repeatability of the LC-MS system, quality control (QC) samples $(n=4)$, which were generated by pooling $10 \mu \mathrm{L}$ aliquots from each embryo extract, were analyzed with the embryo samples.

Chromatography was performed with a Shimadzu Nexera system (Shimadzu; Columbia, MD, USA) coupled to a high-resolution hybrid quadrupole-time-of-flight mass spectrometer (TripleTOF ${ }^{\circledR}$ 5600; SCIEX; 
Framingham, MA, USA). Two different LC analyses using reverse phase and HILIC columns were used. In reverse phase LC, chromatographic separations were carried out using a $4.6 \times 150 \mathrm{~mm}$ Inertsil phenyl-3 column ( $5 \mu \mathrm{m}$, GL Sciences Inc., Rolling Hills Estates, CA, USA) for positive and negative ion analyses, as we described [21]. The sample injection volume was $10 \mu \mathrm{L}$ and the flow rate was $0.4 \mathrm{~mL} / \mathrm{min}$. The mobile phases consisted of water (A) and methanol (B), both with $0.1 \%$ formic acid. The gradient was as follows: an initial hold at $5 \%$ B for $1 \mathrm{~min}$, followed by a gradient of $5-50 \% \mathrm{~B}$ in $11 \mathrm{~min}$, to $100 \% \mathrm{~B}$ at $23 \mathrm{~min}$, held until $35 \mathrm{~min}$, then a shift to $5 \%$ B at $37 \mathrm{~min}$ until $50 \mathrm{~min}$. The column temperature was held at $50{ }^{\circ} \mathrm{C}$. In metabolomics HILIC LC analysis, separation was carried out using a $4.6 \times 150 \mathrm{~mm}$ SeQuant ZICpHILIC ( $5 \mu \mathrm{m}$, EMD Millipore, Billerica, MA, USA). The flow rate was $0.4 \mathrm{~mL} / \mathrm{min}$ and the injection volume was $10 \mu \mathrm{L}$. The two mobile phases consisted of $20 \mathrm{mM}$ ammonium carbonate, $\mathrm{pH} 9.2$ with ammonium hydroxide in water (A) and acetonitrile (B). The gradient was as follows: an initial hold at $80 \%$ B for $1 \mathrm{~min}$, followed by a gradient of $80-20 \%$ B in $30 \mathrm{~min}$, to $8 \%$ B at $31 \mathrm{~min}$, held until $36 \mathrm{~min}$, then a shift to $80 \% \mathrm{~B}$ at $37 \mathrm{~min}$ until $44 \mathrm{~min}$. The column temperature was held at $50{ }^{\circ} \mathrm{C}$.

Time-of-flight (TOF) mass spectrometry (MS) was operated with an acquisition time of $0.25 \mathrm{~s}$ and a scan range of 70-1000 Da. MS/MS acquisition was performed with collision energy set at $35 \mathrm{~V}$ and collision energy spread of $15 \mathrm{~V}$. Each MS/MS scan had an accumulation time of $0.17 \mathrm{~s}$ and a range of 40-1000 Da using information-dependent acquisition (IDA). The source temperature was set at $500{ }^{\circ} \mathrm{C}$ and IonSpray voltage at $4.5 \mathrm{kV}$ in positive ion mode and $-4.0 \mathrm{kV}$ negative ion mode, respectively.

\subsection{Sample preparation, extraction and LC-MS/MS analyses of total or} free DHA, EPA, ARA, and LA fatty acids and hydroxy-DHA

Analysis of total DHA, EPA, ARA, and LA were performed as described [15] with the following modifications: samples were obtained at $24,48,72$, and $120 \mathrm{hpf}(\mathrm{n}=10-15$ embryos per replicate, 3 replicates per group) and saponified in alcoholic $\mathrm{KOH}$ with $1 \%$ ascorbic acid; following cooling, the $\mathrm{pH}$ was adjusted to 2.5 with $12 \mathrm{~N} \mathrm{HCl}$, then $2.0 \mathrm{~mL}$ heptane and $10 \mu \mathrm{L}$ internal standard [DHA- $\mathrm{d}_{5}(1.0 \mu \mathrm{g} / \mathrm{mL})$, EPA$\mathrm{d}_{5}(1.0 \mu \mathrm{g} / \mathrm{mL})$, ARA- $\mathrm{d}_{8}(20.0 \mu \mathrm{g} / \mathrm{mL})$ and LA- $\left.\mathrm{d}_{4}(20.0 \mu \mathrm{g} / \mathrm{mL})\right]$ were added. Samples were mixed, the supernatant (organic layer) was removed and dried under nitrogen gas, the residue resuspended in $100 \mu \mathrm{L} \mathrm{80:20} \mathrm{v/v} \mathrm{methanol:} \mathrm{water} \mathrm{with} 0.5 \%$ acetic acid. Samples were stored at $-80{ }^{\circ} \mathrm{C}$ until analysis by LC-MS/MS (see below).

Extracts for quantitative free fatty acid and hydroxy-DHA analyses were prepared as described for metabolomics samples, with the following modifications: extraction solvent $(290 \mu \mathrm{L}, 80: 20 \mathrm{v} / \mathrm{v}$ methanol:water) included $50 \mu \mathrm{g} / \mathrm{mL}$ BHT and for quantification was combined with $10 \mu \mathrm{L}$ per sample of internal standards containing DHA- $\mathrm{d}_{5}$ $(10.0 \mu \mathrm{g} / \mathrm{mL}), \quad$ EPA- $d_{5} \quad(5.0 \mu \mathrm{g} / \mathrm{mL}), \quad$ ARA-d $8 \quad(20.0 \mu \mathrm{g} / \mathrm{mL}), \quad$ LA- $\mathrm{d}_{4}$ $(20.0 \mu \mathrm{g} / \mathrm{mL})$, and $9(S)-\mathrm{HODE}_{-} \mathrm{d}_{4}(2.0 \mu \mathrm{g} / \mathrm{mL})$. Embryo numbers ranged from $n=15-30$ embryos per replicate, with $n=4$ replicates per group. QC samples were prepared as described for metabolomic analysis. Amounts were quantitated by relative comparison to internal standards, see below.

Chromatographic separations were carried out on $4.6 \times 250 \mathrm{~mm}$ J'sphere ODS-H80 ( $4 \mu \mathrm{m}$, YMC Co, Kyoto, Japan) for negative ion analysis. TOF-MS and TOF-MS/MS were operated with same parameters as for metabolomics, described above. The sample injection volume was $10 \mu \mathrm{L}$ and the flow rate was $1 \mathrm{~mL} / \mathrm{min}$. The mobile phases consisted of water (A) and acetonitrile (B), both with $0.1 \%$ acetic acid. The gradient was as follows: an initial hold at $30 \% \mathrm{~B}$ for $4 \mathrm{~min}$, followed by a gradient of $30-60 \% \mathrm{~B}$ in $1 \mathrm{~min}$, to $65 \% \mathrm{~B}$ at $13 \mathrm{~min}$, to $80 \% \mathrm{~B}$ at $25 \mathrm{~min}$, to $100 \%$ at $26 \mathrm{~min}$ held until $32 \mathrm{~min}$, then a shift to $30 \% \mathrm{~B}$ at $33 \mathrm{~min}$ until $38 \mathrm{~min}$. The column temperature was held at $35^{\circ} \mathrm{C}$.

\subsection{Extracellular flux analyzer assay for bioenergetic profiling}

Oxygen consumption rate (OCR) and proton production rate (PPR) measurements were performed using the XF24 Extracellular Flux Analyzer (Seahorse Bioscience, Billerica, MA) with methods based on [22]. Dual-analyte sensor cartridges were soaked in $500 \mu \mathrm{L}$ XF Calibrant Solution (Seahorse Bioscience, Billerica, MA) in 24-well cell-culture microplates (Seahorse Bioscience, Billerica, MA) overnight at $30^{\circ} \mathrm{C}$ to hydrate. Embryos were staged and placed into 20 of 24 wells on an islet microplate; the remaining four wells served as temperature control wells. Islet plate capture screens were placed over the top of the measurement area to keep the embryos in place. Preliminary experiments were performed to determine the number of embryos needed to achieve OCRs that fell within the recommended specifications of the XF24 instrument for each developmental age: four embryos per well at $24 \mathrm{hpf}$, and two embryos per well at $48 \mathrm{hpf}$. At $24 \mathrm{hpf}$, embryos (4 embryos per well; $\mathrm{n}=7$ wells per VitE condition) were rinsed and placed in unbuffered EM in the XF24 islet capture plate. Briefly, we used oligomycin A to inhibit ADP phosphorylation by the mitochondrial ATP synthase, thereby initiating a decrease in OCR that approximates the fraction of total basal respiration coupled to ATP turnover. Treatment with the uncoupling/protonophore agent, FCCP, causes translocation of protons from the mitochondrial intermembrane space to the matrix, leading to an increase in the OCR that approximates "maximal respiratory capacity". Sodium azide $\left(\mathrm{NaN}_{3}\right)$ blocks the respiratory chain by inhibiting cytochrome c oxidase, leaving "nonmitochondrial respiration". The difference in minimum OCRs measured after oligomycin A and sodium azide treatments is a metric of respiration attributed to proton leak (i.e. ion movements requiring proton motive force) across the mitochondrial inner membrane $[23,24]$. Titrations of each reagent were performed at 24 and $48 \mathrm{hpf}$, to determine the concentrations that produced the maximum change in respiration without inducing death within the experimental time frame. Concentrated stocks of oligomycin and FCCP were prepared in DMSO at $10 \mathrm{mM}$ and $20 \mathrm{mM}$, respectively. A concentrated stock of sodium azide (5 M) was prepared in phosphate-buffered saline.

OCR and PPR were measured before and after the addition of $50 \mu \mathrm{L}$ of $63.2 \mu \mathrm{M}$ oligomycin A (ATP synthase inhibitor), $55.6 \mu \mathrm{L} 2.5 \mu \mathrm{M}$ FCCP (mitochondrial uncoupler), and $61.6 \mu \mathrm{L}$ sodium azide (mitochondrial complex IV inhibitor). The same protocol was used for $48 \mathrm{hpf}$ embryos ( 2 embryos per well; $n=7$ wells per VitE group) with addition of $50 \mu \mathrm{L} 9.2 \mu \mathrm{M}$ oligomycin A; then $56.0 \mu \mathrm{L} 2.8 \mu \mathrm{M}$ FCCP; then $62 \mu \mathrm{L}$ $1.25 \mathrm{mM} \mathrm{NaN}_{3}$. The Seahorse protocol consisted of calibration, equilibration, 8 measurements of baseline, 8 measurements after injection of oligomycin A and FCCP, and 12 measurements after injection of $\mathrm{NaN}_{3}$. OCRs and PPRs were calculated using a modified AKOS algorithm, available with the Seahorse Bioscience software, that takes into account oxygen diffusion through the plate and atmospheric leak, in addition to the oxygen consumed by the organism [22]. Buffer capacity was calculated by monitoring $\mathrm{pH}$ of the media, and PPR values derived based on the calculated buffer capacity and chamber volume of each well.

\subsection{Microinjection rescue studies}

Embryos were microinjected using a nanoliter 2000 injector (World Precision Instruments, Sarasota, FL, USA) linked to a stereoscopic microscope. Needles for microinjection were made in a puller (Sutter Instruments, model P-97, Novato, CA, USA) using glass capillary with an internal filament (1.14 o.d.; $0.5 \mathrm{~mm}$ i.d.). For pilot $\alpha$-tocopherol injections, embryos were injected at the 1-2 cell stage (approximately $0.5 \mathrm{hpf}$ according to [11]) into the yolk-sac with a sterile vitamin $\mathrm{E}$ preparation made for intravenous use in humans containing $d_{6}-\alpha$ tocopherol $\left(\mathrm{d}_{6}-\alpha-\mathrm{T}, 5.4 \mathrm{~g} / \mathrm{L}\right)$ in an oil-water emulsion made with soybean oil and phospholipids. Embryos were injected with $4.0 \mathrm{~nL}$ of the $d_{6}-\alpha-T$ emulsion, a quantity ( $20 \mathrm{ng}$, or $46 \mathrm{pmol}$ ) that provides an 

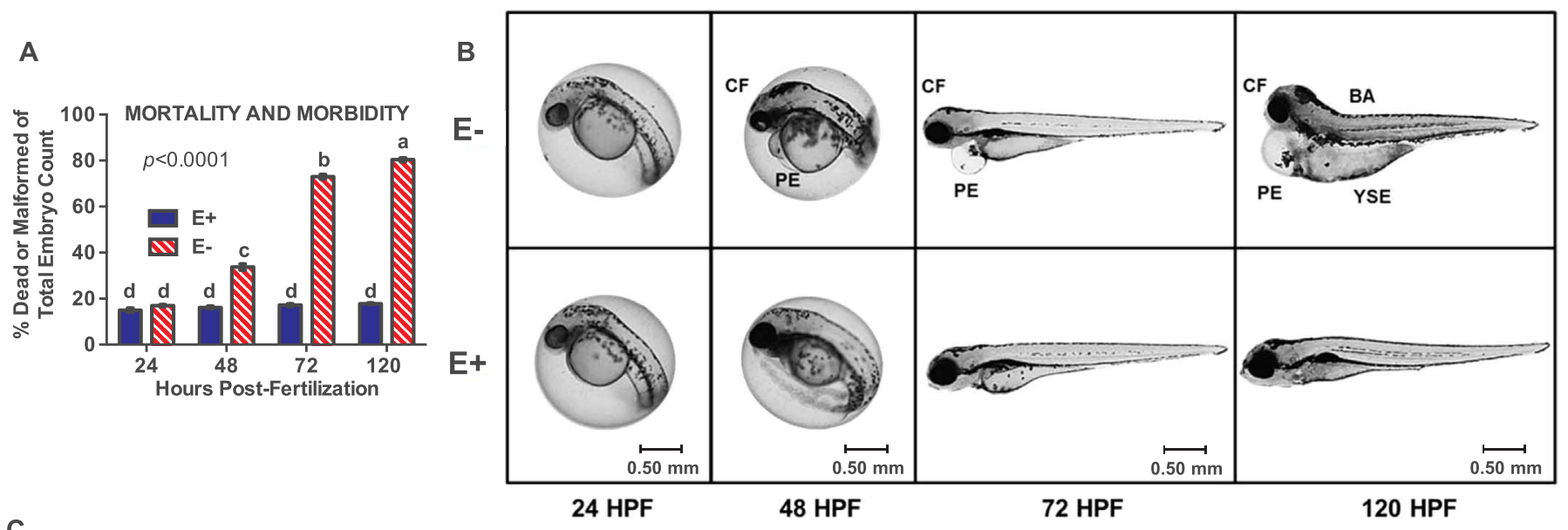

C
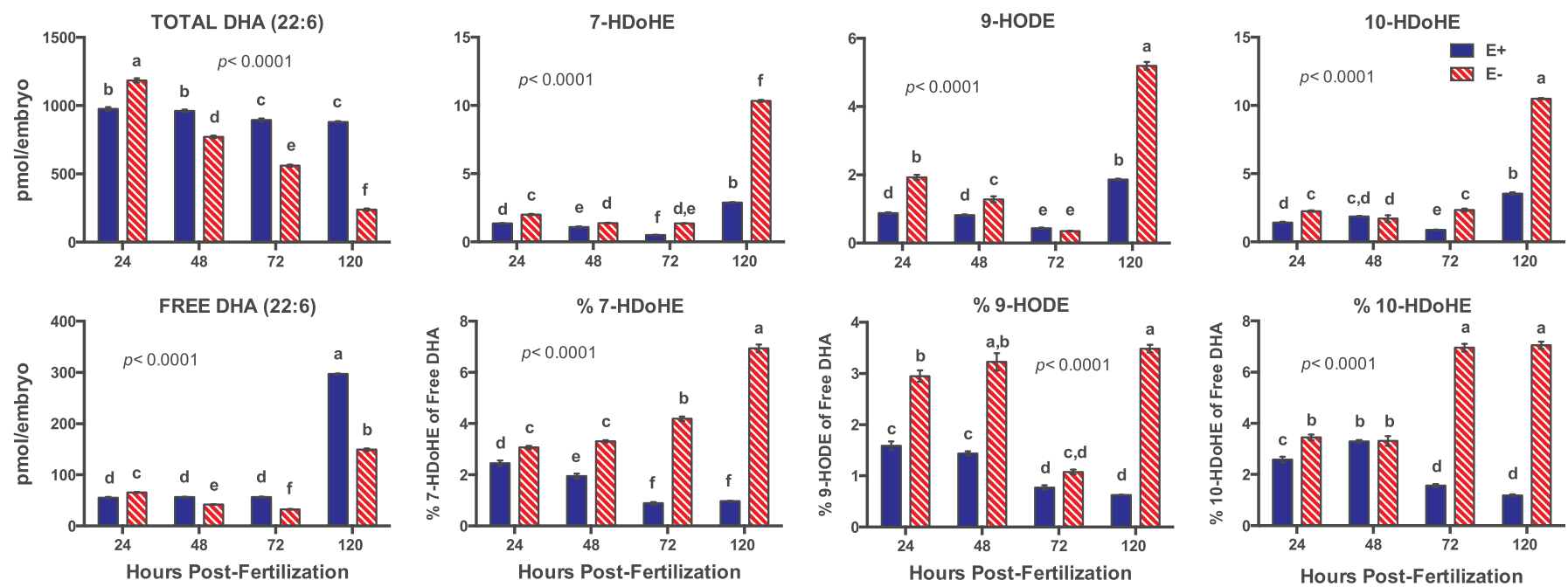

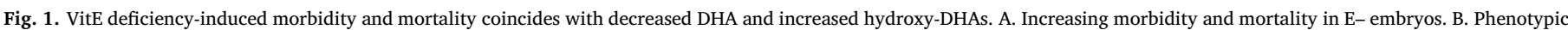

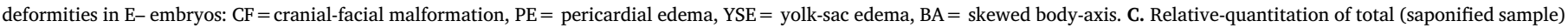

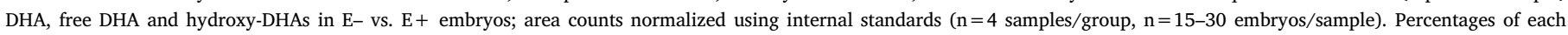
hydroxy-DHA $=($ hydroxy-DHA/free DHA)*100. Shown are means \pm SEM; $p$-values from 2-way ANOVA with unique letters indicating differences (Tukey's post-test, $p<0.05)$.

amount of $\alpha$-tocopherol to the E- embryos equal to that in $0 \mathrm{hpf} \mathrm{E}+$ embryos [15]. Glucose injections were performed as described [25]. Briefly, at 24 hpf embryos were injected (into the yolk-sac) with $4.0 \mathrm{~nL}$ of 2.0 M glucose solution prepared with $D$-glucose (Sigma-Aldrich, St Louis, MO, USA) dissolved in sterile saline solution. This dose was based on the maximum solubility of glucose in water. Control-injection conditions for both experiments consisted of saline injection with sterile saline solution ( $\mathrm{NaCl}, 58 \mathrm{mmol} / \mathrm{L} ; \mathrm{KCl}, 0.7 \mathrm{mmol} / \mathrm{L} ; \mathrm{MgSO}_{4}, 0.4 \mathrm{mmol} /$ $\left.\mathrm{L} ; \mathrm{Ca}\left(\mathrm{NO}_{3}\right)_{2}, 0.6 \mathrm{mmol} / \mathrm{L} ; \mathrm{Hepes}, 5 \mathrm{mmol} / \mathrm{L} ; \mathrm{pH} 7.3\right)$. Criteria used to assess supplementation tolerance of zebrafish embryos using ZAAP were embryonic development, growth, and mortality, assessed at 24, 48, and $120 \mathrm{hpf}$.

\subsection{Data processing and statistical analyses}

Targeted metabolomics data processing was performed using PeakView software (SCIEX). Sample peaks for each targeted metabolite of interest were annotated using the extracted ion chromatograms (XIC) lists based on high resolution MS, MS/MS fragmentation, isotopic distribution, and retention time compared with an in-house library of 635 metabolite standards (IROA Technologies, Bolton, MA, USA). In addition to the IROA database, metabolite identities were confirmed further using the METLIN web-based metabolomics database (http:// www.metlin.scripps.edu). Peak intensities for each individual metabolic feature were normalized using the corresponding mean QC sample $(n=4)$ intensity for that feature, as described [26], to balance their differences in intensities that may have arisen due to discrepancies in the sample homogenization (sample preparation). Student's $t$-tests (Excel, Microsoft) to compare the two VitE groups at each developmental time-point (24, 48, 72, and $120 \mathrm{hpf}$ ) were performed with statistical significance set at $p<0.05$. To control for false discovery rates for these metabolomics data during follow-up analyses, study outcome significance was set at $Q<0.05$, using an adaptive linear step-up procedure [27]. Subsequent statistical analyses (e.g. 2-way ANOVA with Tukey's or Sidak's multiple comparison tests, as recommended by the software) were performed using GraphPad Prism 6.0 software (GraphPad, La Jolla, CA).

Quantification of total and free fatty acids, and hydroxy-DHA was performed using MutliQuant Software version 3.0.2 (SCIEX). Lipid peaks were identified based on the accurate masses and retention times of each individual lipid, then quantified by integrating peak area using MultiQuant Software. Raw area counts for each lipid were normalized using area counts for the corresponding internal standard, then corrected for internal standard concentration. Statistical analyses (e.g. 2-way ANOVA with Tukey's multiple comparison test) were performed using GraphPad Prism 6.0 software (GraphPad, La Jolla, CA).

For OCR analyses, the extracellular flux analyzer assay data was collected and processed using the XF Reader software (Seahorse Biosciences, Agilent Technologies, Santa Clara, CA) and exported using Excel 2013 (Microsoft). The baseline OCR and PPR were calculated, in 


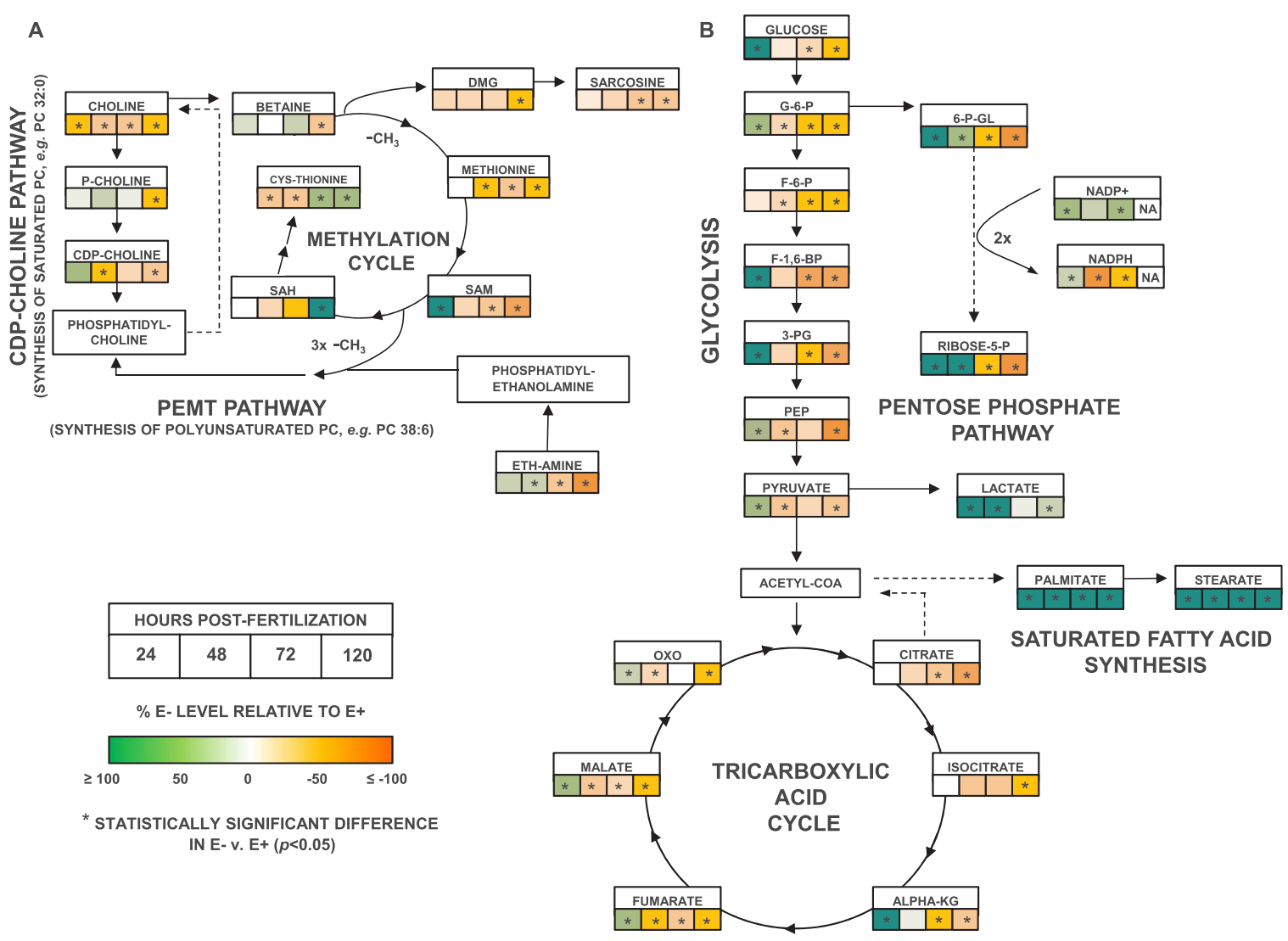

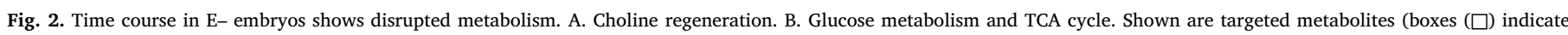

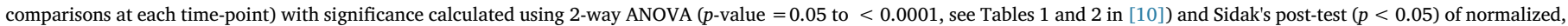

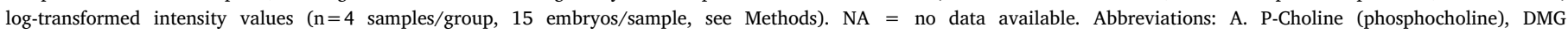

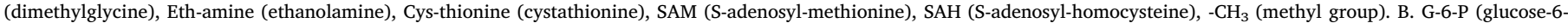

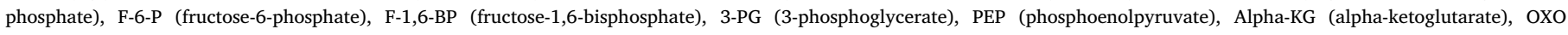
(oxaloacetate), 6-P-GL (6-phosphogluconate), Ribose 5-P (ribose-5-phosphate).

addition to the ATP-linked OCR, the maximum OCR, proton leak, and mitochondrial reserve capacity, as described [22,23]. Additional statistical analyses (e.g. 2-way ANOVA with Tukey's multiple comparison tests) were performed using GraphPad Prism 6.0 software.

Statistical analyses for morphological and behavioral endpoints were performed using code developed in $\mathrm{R}$ ( $\mathrm{R}$ Developmental Core Team 2014, http://www.R-project.org). For morphological assessments at 24 and $96 \mathrm{hpf}$, binary responses were recorded as either absent (0) or present (1) for each of the 18 endpoints. For statistical analyses of behavior, raw data files were processed using custom $\mathrm{R}$ scripts with methodologies based on [19]. In brief, the distance traveled by each individual animal, over each integration period, was measured and then the total area under the curve (AUC) was computed for each animal. The overall AUCs for the E- compared to the E + embryos then were compared using a combination of percent change (minimum $30 \%$ difference from $\mathrm{E}+$ embryos) and a Kolmogorov-Smirnov test $(p<0$. $01)$ to determine statistical significance.

\section{Results and discussion}

We obtained E- and E + embryos by spawning adult 5D zebrafish fed either E- or E+ diets. E+ embryos grew normally, while Eembryos suffered $>80 \%$ morbidity and mortality by 120 hpf (Fig. 1AB). Measured $\alpha$-tocopherol concentrations in living E- and E + embryos were $1.09 \pm 0.01$ and $22.41 \pm 0.04 \mathrm{pmol} / \mathrm{embryo}$, respectively, at 120 hpf.

At 48 hpf E- embryos contained decreased concentrations of DHAcontaining phospholipids (DHA-PL), particularly phosphatidylcholine
(PC) and phosphatidylethanolamine (PE), as well as DHA-lyso-PC [28], a major DHA transporter to the brain $[29,30]$. Since DHA is critical for normal neurodevelopment [29], we hypothesized that VitE deficiency causes embryonic death by depleting DHA-PC, thereby depriving the embryo of adequate DHA. We tested this hypothesis by assessing the impact of VitE status on relative quantities of total, free (unesterified) and oxidized DHA in E- vs. E + embryos. Initially, total and free DHA were elevated in E- embryos, but by $48 \mathrm{hpf}$ both were significantly lower than E+ embryos (Fig. 1C, see also Fig. 1 and Tables 1 and 2 in Supplementary data); further, the lipid peroxidation products, 7- and 10-hydroxy-DHA [31], as well as 9-HODE, were elevated in E(Fig. 1C), ranging from $3 \%$ to $7 \%$ of the free DHA at $120 \mathrm{hpf}$, confirming increased DHA peroxidation.

Craniofacial malformations are observed at $48 \mathrm{hpf}$ in E- embryos (Fig. 1B) and in zebrafish embryos genetically manipulated to completely lack VitE [32] more severe defects are seen as early as 15-17 hpf, an embryonic stage that coincides with neurulation [11]. Since choline is a nutrient essential for neurulation in vertebrate embryos [33] and PC is a major source of choline [34], we hypothesized that the increased DHA-PC replenishment in E- embryos [28] induces a secondary choline deficiency. We found that as development progressed both choline and various choline-containing compounds in the cytidine-5-diphosphocholine (CDP-choline) pathway for PC synthesis decreased in the Eembryos (Fig. 2A; see also Fig. 2, Tables 1 and 2 in Supplementary data), consistent with our previous lipidomics-based investigations [35].

PC can also be generated by the serial methylation of PE via the phosphatidylethanolamine N-methyltransferase (PEMT) pathway using 

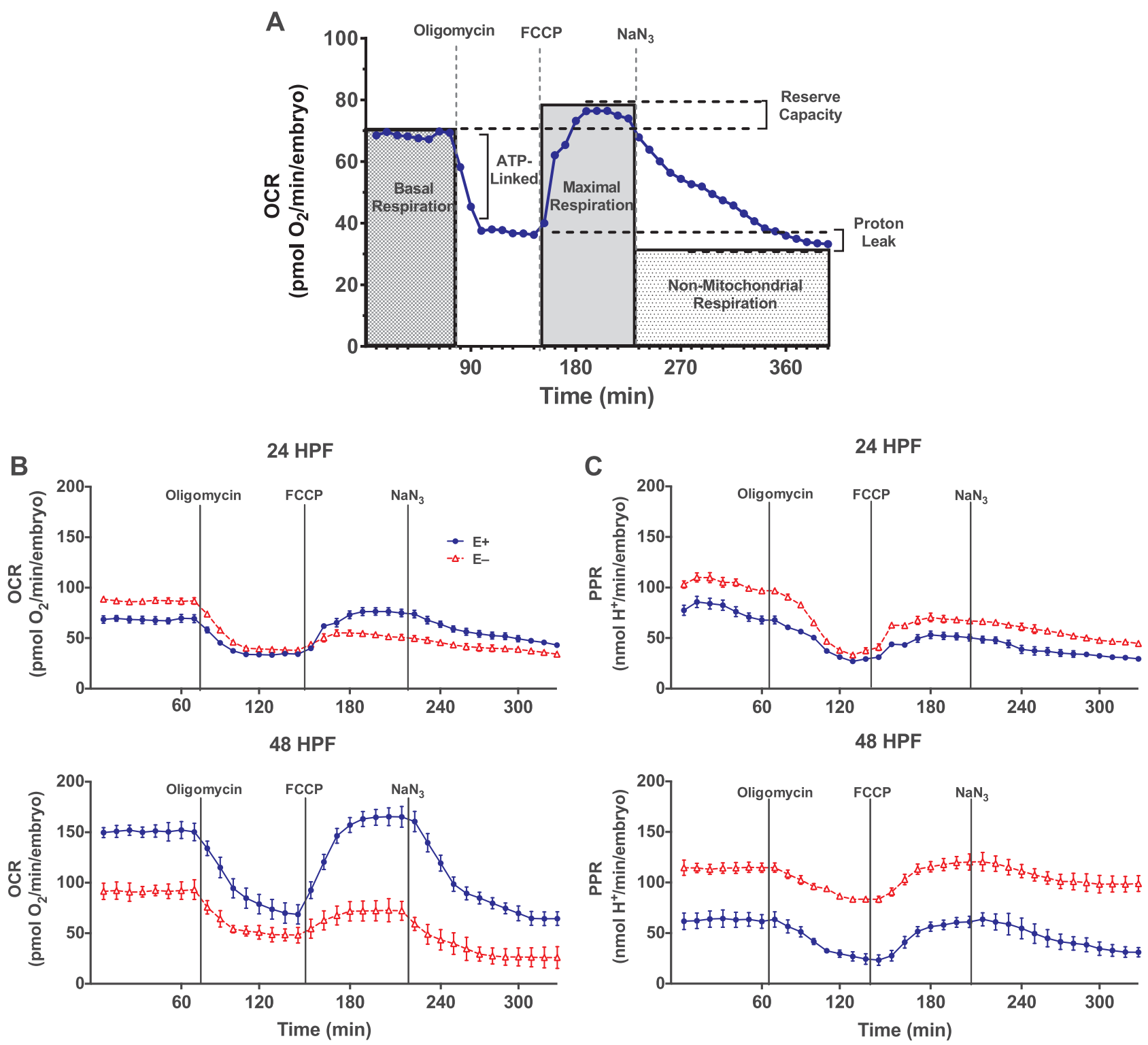

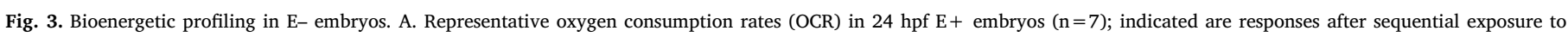

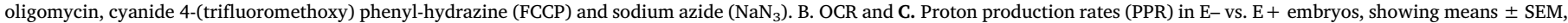
$\mathrm{n}=7$ samples/group; for 24 hpf: 4 embryos/sample for 36/44 trials; for 48 hpf: 2 embryos/sample, 36/36 trials.

the requisite methyl-donor, S-adenosylmethionine (SAM). Unlike the CDP-choline pathway that generates PC containing mono- and diunsaturated fatty acids, the PEMT pathway generates PC containing polyunsaturated fatty acids [36] and enhances DHA transfer from mother to fetus in humans [37]. Evidence that the PEMT pathway in E- embryos caused depletion of methyl-donors is shown by the decreases at $120 \mathrm{hpf}$ of SAM, methionine, and betaine, while the SAM oxidation product, S-adenosyl-homocysteine increased (Fig. 2A; see also Fig. 2, Tables 1 and 2 in Supplementary data). Thus, PC synthesis via the PEMT pathway in E- embryos became compromised due to choline and methyl-donor depletion.

VitE deficiency not only induced oxidative damage by increasing lipid peroxidation, but also depleted other antioxidants (see also Fig. 3, Tables 1 and 2 in Supplementary data). Specifically measured ascorbic acid concentrations in E- embryos were halved at $120 \mathrm{hpf}(22.2 \pm 0.3$ vs. $59.8 \pm 1.0$ pmol/embryo in E- and E+, respectively, see also Fig. 3, Tables 1 and 2 in Supplementary data). We, therefore, hypothesized that the E- embryos have an increased need for reducing equivalents (NADPH) and that glucose would be shunted to the pentose phosphate pathway to generate NADPH [38]. In E- embryos, glucose was depleted with increases in 6-phosphogluconate and ribose-5-phosphate intermediates (Fig. 2B), while glycolytic and TCA cycle intermediates in Eembryos (although increased at $24 \mathrm{hpf}$ ) were decreased at subsequent time-points (Fig. 2B; see also Fig. 4, Tables 1 and 2 in Supplementary data). Moreover, NADPH was increasingly oxidized in E- embryos and $\mathrm{NADP}+/ \mathrm{NADPH}$ ratios increased over time (Fig. 2B; see also Fig. 3, Tables 1 and 2 in Supplementary data). This metabolic shift - aimed at ameliorating the effects of oxidative stress - occurred at the expense of energy-generating pathways, such as glycolysis and flow through the respiratory chain of tricarboxylic acid (TCA) cycle-generated reducing equivalents. Evidence for the uncoupling of the TCA cycle in Eembryos (i.e. citrate transported to cytosol for fatty acid synthesis and cytosolic reduction of pyruvate to lactate) is provided by their 1) increased levels of saturated fatty acids (palmitic and stearic, Fig. 2B, see also Figure 5, Tables 1 and 2 in Supplementary data), 2) decreased glutamine and glutamate (see also Tables 1 and 2 in Supplementary data), and 3) increased lactate (Fig. 2B, see also Fig. 4, Tables 1 and 2 in Supplementary data). Overall, this metabolic profile in E- embryos resembles the Warburg effect seen in cancer cells [39]. Thus, Eembryos experience dysregulation of energy metabolism, a phenomen- 

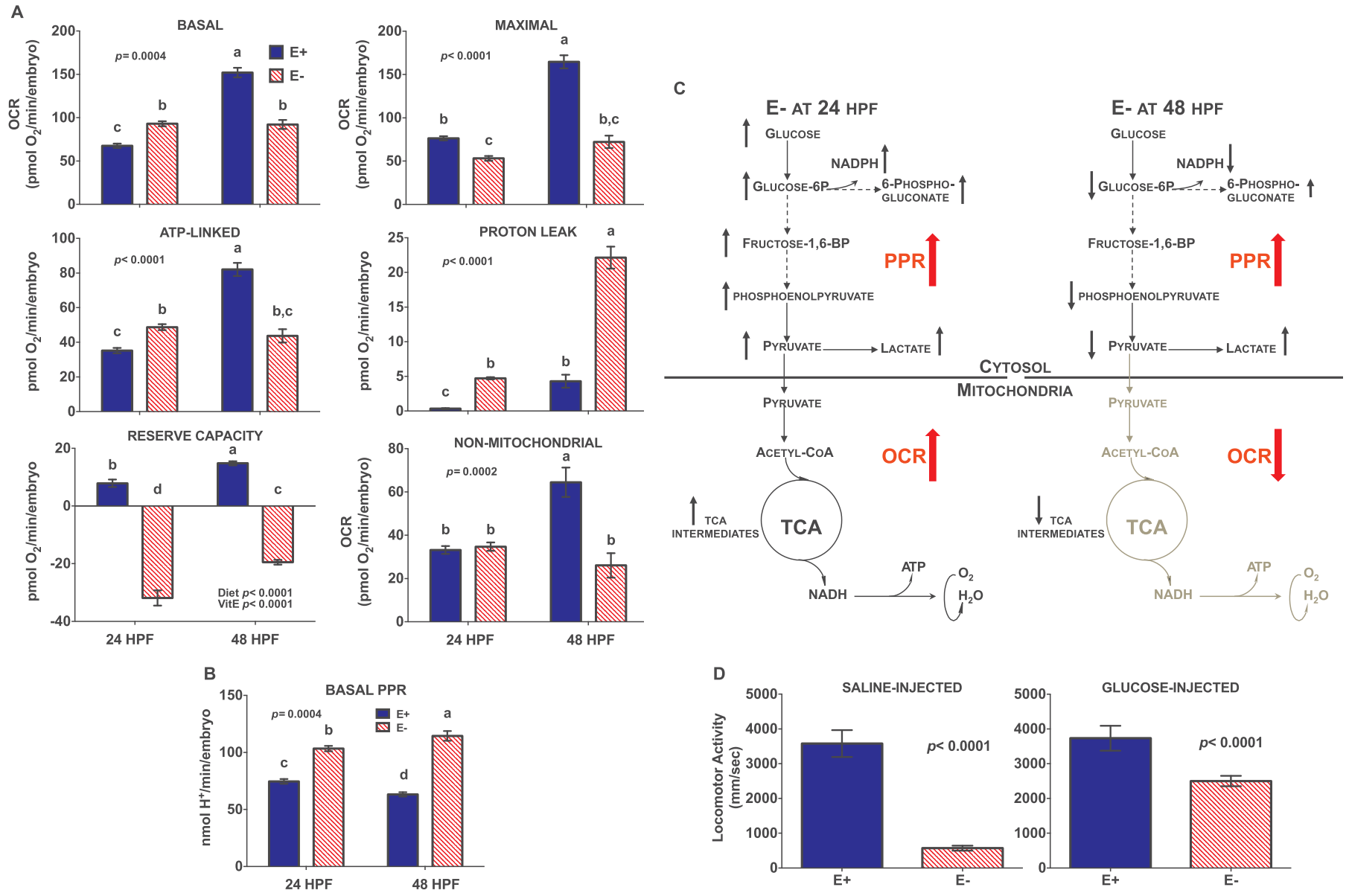
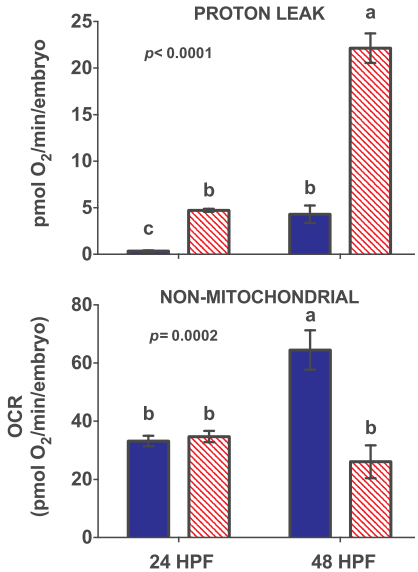
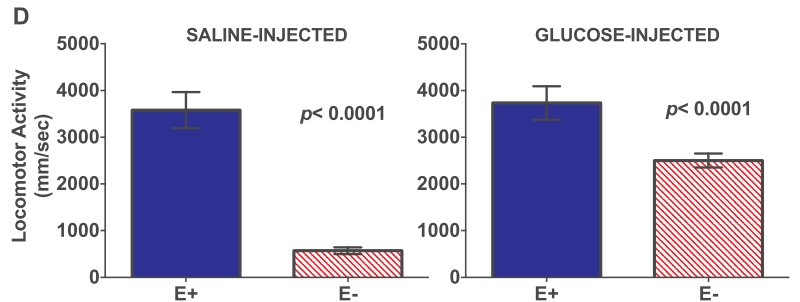

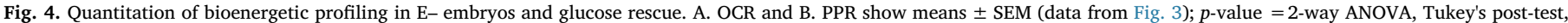

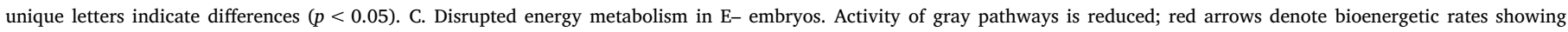

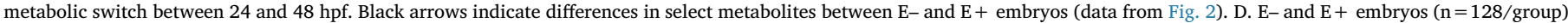

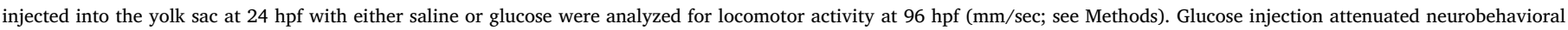
abnormalities in E- embryos by $\sim 50 \%$ (Kolmogorov-Smirnov test; $p<0.0001$ ).

on that is a major driver of cellular dysfunction and death as an ultimate consequence of VitE deficiency. Potentially, ferroptosis, a mechanism of programmed cell death due to increased lipid peroxidation $[40,41]$, which is dependent on GPx4 and GSH, as well as VitE to prevent cell death [7], is responsible for the embryonic lethality. Notably, VitE has been suggested to function as a lipoxygenase inhibitor and thereby prevents the generation of oxidized arachidonic and adrenic PE, which have been identified as lipid signaling molecules for ferroptosis [42]. These latter molecules were doubly and triply oxygenated, and thus beyond the technical capabilities of the approaches used for the present study, so further research is needed to assess the role of ferroptosis during VitE deficiency and embryogenesis in zebrafish. Additionally, endothelium specific Gpx4 depletion in mice showed that VitE was necessary not only for endothelial function but also mouse viability [43]. Moreover, studies in mice with a Gpx4 deletion in hematopoietic cells showed that GPx4 is essential for preventing receptor interacting protein 3 (RIP3)-dependent necroptosis in erythroid precursor cells, a process that could be ameliorated by VitE [44]. Thus, there are a number of pathways involving lipid peroxidation, GPx4 and VitE that lead to programmed cell death and emphasize the necessity for further studies to elucidate these various mechanisms.

To assess the degree to which energy metabolism was dysregulated during VitE deficiency, the bioenergetic profiles of E- and E + embryos were assessed in real-time by measuring mitochondrial and nonmitochondrial respiration (Fig. 3A). At 24 hpf, E- embryos had higher basal oxygen consumption rates (OCR), but at $48 \mathrm{hpf}$ their basal OCRs were decreased (Fig. 3B). At 24 and $48 \mathrm{hpf}$ E- embryos had reduced levels of "maximal respiration" (Figs. 3B, 4A), demonstrating a lack of mitochondrial reserve capacity [23], and had an elevated proton production rates (PPR, Figs. 3C, 4B), concomitant with their increased lactate concentrations (Fig. 2B). Thus, E- embryos experienced a "metabolic switch" from a hyper- to a hypo-metabolic state consistent with non-energy generating glucose metabolism (i.e. pentose phosphate pathway) and TCA cycle uncoupling (i.e. citrate transport into cytosol) by 48 hpf (Figs. 2B, 4C).

We hypothesized that impairment of aerobic, mitochondrial glucose metabolism in E- embryos caused their neurobehavioral abnormalities. Morphologically normal $96 \mathrm{hpf}$ E- embryos were 84\% less responsive to light-dark stimuli in a locomotor assay (Fig. 4D; see also Figure 6 in Supplementary data). To determine if increased glucose status would ameliorate the neurobehavioral defects, 24 hpf E- and E + embryos were microinjected into the yolk sac with $\mathrm{D}-(+)$-glucose in saline or with saline alone. Glucose rescued the neurological deficits in $50 \%$, and morbidity and mortality in 31\%, of E- embryos (Fig. 4D; see also Figure 7 in Supplementary data), but they still sustained craniofacial deformities (not shown). Thus, it may be surmised that other nutrients, e.g. DHA and choline, are required by E- embryos to obtain a complete phenotypic rescue, as observed in a pilot trial employing microinjection of a VitE-PL emulsion at 0 hpf (see Figure 6 in Supplementary data). Deuterated polyunsaturated fatty acids, which are less susceptible to lipid peroxidation [45], and protect mitochondria from dysregulation caused by lipid peroxidation [46] could be used as rescue reagents in $\mathrm{E}-$ embryos to evaluate the role of the initial lipid peroxidation in embryonic lethality. Similarly, ferroptosis inhibitors, 
such as ferrostatin and liproxstatin [47], might be protective during VitE deficiency and would be a means to test directly the role of ferroptosis in E- embryo lethality. Clearly, further study is needed to define the molecular mechanisms by which VitE deficiency becomes lethal.

The mechanisms underlying VitE's essentiality during neurodevelopment include its antioxidant protection of DHA and DHA-PLs, which prevents lipid peroxidation, additional antioxidant depletion, and metabolic dysregulation with secondary decreases in both choline and glucose. Additionally, specific effects on lipoxygenase enzymes may play critical roles in embryonic lethality. Such pleiotropic outcomes cause death by effectively starving the embryonic brain not only of vital nutrients, namely DHA and choline, but also of the energy needed for development. These findings acquire even greater significance upon consideration that - nearly 100 years after its discovery [1] - they provide mechanistic insights into why the vertebrate fetus requires VitE, and, as a corollary, expand the role VitE deficiency plays in human miscarriage.

\section{Acknowledgements}

The authors thank Carrie Barton, Greg Gonnerman, Andrea Knecht, Jane La Du, Scott Leonard, and Lisa Truong for providing outstanding technical assistance. Tory Hagen, Siva Kolluri and Andrew Karplus are gratefully acknowledged for reading the manuscript and providing critical advice. National Institutes of Health Grants S10RR027878 (MGT and JFS) and NIEHS P30 ES000201 (RT) supported this work. MM was supported in part by National Science Foundation Grant DGE 0965820. H-KK sabbatical support provided by The Catholic University of Korea. MGT supported in part by the Helen P Rumbel endowment to the LPI.

\section{Appendix A. Supplementary material}

Supplementary material associated with this article can be found in the online version at doi:10.1016/j.freeradbiomed.2017.01.020 or see [10].

\section{References}

[1] H.M. Evans, K.S. Bishop, On the existence of a hitherto unrecognized dietary factor essential for reproduction, Science 56 (1922) 650-651.

[2] G.W. Burton, A. Joyce, K.U. Ingold, First proof that vitamin E is major lipid-soluble, chain-breaking antioxidant in human blood plasma, Lancet 2 (1982) 327.

[3] R. Zanetti, A. Catala, Changes in n-6 and n-3 polyunsaturated fatty acids during lipid-peroxidation of mitochondria obtained from rat liver and several brain regions: effect of alpha-tocopherol, Prostaglandins Leukot. Ess. Fat. Acids 62 (2000) 379-385.

[4] B.A. Wagner, G.R. Buettner, C.P. Burns, Free radical-mediated lipid peroxidation in cells: oxidizability is a function of cell lipid bis-allylic hydrogen content, Biochemistry 33 (1994) 4449-4453.

[5] E. Cadenas, L. Packer, M.G. Traber, Antioxidants, oxidants, and redox impacts on cell function - A tribute to Helmut Sies, Arch. Biochem. Biophys. 595 (2016) 94-99.

[6] F. Ursini, M. Maiorino, M. Valente, L. Ferri, C. Gregolin, Purification from pig liver of a protein which protects liposomes and biomembranes from peroxidative degradation and exhibits glutathione peroxidase activity on phosphatidylcholine hydroperoxides, Biochim. Biophys. Acta 710 (1982) 197-211.

[7] B.A. Carlson, R. Tobe, E. Yefremova, P.A. Tsuji, V.J. Hoffmann, U. Schweizer, V.N. Gladyshev, D.L. Hatfield, M. Conrad, Glutathione peroxidase 4 and vitamin E cooperatively prevent hepatocellular degeneration, Redox Biol. 9 (2016) 22-31.

[8] A.A. Shamim, K. Schulze, R.D. Merrill, A. Kabir, P. Christian, S. Shaikh, L. Wu, H. Ali, A.B. Labrique, S. Mehra, R.D. Klemm, M. Rashid, P. Sungpuag, E. Udomkesmalee, K.P. West Jr., First-trimester plasma tocopherols are associated with risk of miscarriage in rural Bangladesh, Am. J. Clin. Nutr. 101 (2015) 294-301.

[9] P. Szabolcs, F. Angelika, F.F. Roos, A. Wyss, M. Eggersdorfer, K. Hoffmann, P. Weber, A systematic review of global alpha-tocopherol status as assessed by nutritional intake levels and blood serum concentrations, Int. J. Vitam. Nutr. Res. (2016) 1-21.

[11] C.B. Kimmel, W.W. Ballard, S.R. Kimmel, B. Ullmann, T.F. Schilling, Stages of embryonic development of the zebrafish, Dev. Dyn. 203 (1995) 253-310.

[12] K.M. Lebold, D.B. Jump, G.W. Miller, C.L. Wright, E.M. Labut, C.L. Barton, R.L. Tanguay, M.G. Traber, Vitamin E deficiency decreases long-chain PUFA in zebrafish (Danio rerio), J. Nutr. 141 (2011) 2113-2118.

[13] G.W. Miller, E.M. Labut, K.M. Lebold, A. Floeter, R.L. Tanguay, M.G. Traber, Zebrafish (Danio rerio) fed vitamin E-deficient diets produce embryos with increased morphologic abnormalities and mortality, J. Nutr. Biochem. 23 (2012) 478-486.

[14] M. Westerfield, The Zebrafish Book; A guide for the Laboratory Use of Zebrafish (Danio rerio), University of Oregon Press, Eugene, 2007.

[15] K.M. Lebold, J.S. Kirkwood, A.W. Taylor, J. Choi, C.L. Barton, G.W. Miller, J. La Du, D.B. Jump, J.F. Stevens, R.L. Tanguay, M.G. Traber, Novel liquid chromatographymass spectrometry method shows that vitamin E deficiency depletes arachidonic and docosahexaenoic acids in zebrafish (Danio rerio) embryos, Redox Biol. 2 (2013) 105-113.

[16] B. Frei, L. England, B.N. Ames, Ascorbate is an outstanding antioxidant in human blood plasma, Proc. Natl. Acad. Sci. USA 86 (1989) 6377-6381.

[17] K.M. Lebold, C.V. Lohr, C.L. Barton, G.W. Miller, E.M. Labut, R.L. Tanguay, M.G. Traber, Chronic vitamin E deficiency promotes vitamin C deficiency in zebrafish leading to degenerative myopathy and impaired swimming behavior, Comp. Biochem. Physiol. C Toxicol. Pharm. 157 (2013) 382-389.

[18] K.S. Saili, M.M. Corvi, D.N. Weber, A.U. Patel, S.R. Das, J. Przybyla, K.A. Anderson, R.L. Tanguay, Neurodevelopmental low-dose bisphenol A exposure leads to early life-stage hyperactivity and learning deficits in adult zebrafish, Toxicology 291 (2012) 83-92.

[19] P.D. Noyes, D.E. Haggard, G.D. Gonnerman, R.L. Tanguay, Advanced morphological - behavioral test platform reveals neurodevelopmental defects in embryonic zebrafish exposed to comprehensive suite of halogenated and organophosphate flame retardants, Toxicol. Sci. 145 (2015) 177-195.

[20] L. Truong, K.S. Saili, J.M. Miller, J.E. Hutchison, R.L. Tanguay, Persistent adult zebrafish behavioral deficits results from acute embryonic exposure to gold nanoparticles, Comp. Biochem. Physiol. C Toxicol. Pharm. 155 (2012) 269-274.

[21] J.S. Kirkwood, K.M. Lebold, C.L. Miranda, C.L. Wright, G.W. Miller, R.L. Tanguay, C.L. Barton, M.G. Traber, J.F. Stevens, Vitamin C deficiency activates the purine nucleotide cycle in zebrafish, J. Biol. Chem. 287 (2012) 3833-3841.

[22] K.D. Stackley, C.C. Beeson, J.J. Rahn, S.S. Chan, Bioenergetic profiling of zebrafish embryonic development, PLoS One 6 (2011) e25652.

[23] B.P. Dranka, G.A. Benavides, A.R. Diers, S. Giordano, B.R. Zelickson, C. Reily, L. Zou, J.C. Chatham, B.G. Hill, J. Zhang, A. Landar, V.M. Darley-Usmar, Assessing bioenergetic function in response to oxidative stress by metabolic profiling, Free Radic. Biol. Med. 51 (2011) 1621-1635.

[24] B.G. Hill, G.A. Benavides, J.R. Lancaster Jr., S. Ballinger, L. Dell'Italia, Z. Jianhua, V.M. Darley-Usmar, Integration of cellular bioenergetics with mitochondrial quality control and autophagy, Biol. Chem. 393 (2012) 1485-1512.

[25] F. Rocha, J. Dias, S. Engrola, P. Gavaia, I. Geurden, M.T. Dinis, S. Panserat, Glucose overload in yolk has little effect on the long-term modulation of carbohydrate metabolic genes in zebrafish (Danio rerio), J. Exp. Biol. 217 (2014) 1139-1149.

[26] M.R. Elie, J. Choi, Y.M. Nkrumah-Elie, G.D. Gonnerman, J.F. Stevens, R.L. Tanguay, Metabolomic analysis to define and compare the effects of PAHs and oxygenated PAHs in developing zebrafish, Environ. Res. 140 (2015) 502-510.

[27] Y. Benjamini, A.M. Krieger, D. Yekutieli, Adaptive linear step-up procedures that control the false discovery rate, Biometrika 93 (2006) 491-507.

[28] M.Q. McDougall, J. Choi, J.F. Stevens, L. Truong, R.L. Tanguay, M.G. Traber, Lipidomics and H2(18)O labeling techniques reveal increased remodeling of DHAcontaining membrane phospholipids associated with abnormal locomotor responses in alpha-tocopherol deficient zebrafish (danio rerio) embryos, Redox Biol. 8 (2016) 165-174.

[29] V. Alakbarzade, A. Hameed, D.Q. Quek, B.A. Chioza, E.L. Baple, A. CazenaveGassiot, L.N. Nguyen, M.R. Wenk, A.Q. Ahmad, A. Sreekantan-Nair, M.N. Weedon, P. Rich, M.A. Patton, T.T. Warner, D.L. Silver, A.H. Crosby, A partially inactivating mutation in the sodium-dependent lysophosphatidylcholine transporter MFSD2A causes a non-lethal microcephaly syndrome, Nat. Genet. 47 (2015) 814-817.

[30] A. Guemez-Gamboa, L.N. Nguyen, H. Yang, M.S. Zaki, M. Kara, T. Ben-Omran, N. Akizu, R.O. Rosti, B. Rosti, E. Scott, J. Schroth, B. Copeland, K.K. Vaux, A. Cazenave-Gassiot, D.Q. Quek, B.H. Wong, B.C. Tan, M.R. Wenk, M. Gunel, S. Gabriel, N.C. Chi, D.L. Silver, J.G. Gleeson, Inactivating mutations in MFSD2A, required for omega-3 fatty acid transport in brain, cause a lethal microcephaly syndrome, Nat. Genet. 47 (2015) 809-813.

[31] P.B. Derogis, F.P. Freitas, A.S. Marques, D. Cunha, P.P. Appolinario, F. de Paula, T.C. Lourenco, M. Murgu, P. Di Mascio, M.H. Medeiros, S. Miyamoto, The development of a specific and sensitive LC-MS-based method for the detection and quantification of hydroperoxy- and hydroxydocosahexaenoic acids as a tool for lipidomic analysis, PLoS One 8 (2013) e77561.

[32] G.W. Miller, L. Ulatowski, E.M. Labut, K.M. Lebold, D. Manor, J. Atkinson, C.L. Barton, R.L. Tanguay, M.G. Traber, The alpha-tocopherol transfer protein is essential for vertebrate embryogenesis, PLoS One 7 (2012) e47402.

[33] M.C. Fisher, S.H. Zeisel, M.H. Mar, T.W. Sadler, Perturbations in choline metabolism cause neural tube defects in mouse embryos in vitro, FASEB J. 16 (2002) 619-621.

[34] Z. Li, D.E. Vance, Phosphatidylcholine and choline homeostasis, J. Lipid Res. 49 (2008) 1187-1194.

[35] J. Choi, S.W. Leonard, K. Kasper, M. McDougall, J.F. Stevens, R.L. Tanguay, M.G. Traber, Novel function of vitamin E in regulation of zebrafish (Danio rerio) brain lysophospholipids discovered using lipidomics, J. Lipid Res. 56 (2015) $1182-1190$.

[36] C.J. Pynn, N.G. Henderson, H. Clark, G. Koster, W. Bernhard, A.D. Postle, Specificity and rate of human and mouse liver and plasma phosphatidylcholine synthesis analyzed in vivo, J. Lipid Res. 52 (2011) 399-407.

[37] J. Yan, X. Jiang, A.A. West, C.A. Perry, O.V. Malysheva, J.T. Brenna, S.P. Stabler, 
R.H. Allen, J.F. Gregory 3rd, M.A. Caudill, Pregnancy alters choline dynamics: results of a randomized trial using stable isotope methodology in pregnant and nonpregnant women, Am. J. Clin. Nutr. 98 (2013) 1459-1467.

[38] B.L. Bartnik, S.M. Lee, D.A. Hovda, R.L. Sutton, The fate of glucose during the period of decreased metabolism after fluid percussion injury: a ${ }^{13} \mathrm{C}$ NMR study, $\mathrm{J}$ Neurotrauma 24 (2007) 1079-1092.

[39] S. Devic, Warburg effect - a consequence or the cause of carcinogenesis?, J. Cancer 7 (2016) 817-822.

[40] H. Yu, P. Guo, X. Xie, Y. Wang, G. Chen, Ferroptosis, a new form of cell death, and its relationships with tumourous diseases, J. Cell Mol. Med. (2016).

[41] S. Tuzlak, T. Kaufmann, A. Villunger, Interrogating the relevance of mitochondrial apoptosis for vertebrate development and postnatal tissue homeostasis, Genes Dev. 30 (2016) 2133-2151.

[42] V.E. Kagan, G. Mao, F. Qu, J.P. Angeli, S. Doll, C.S. Croix, H.H. Dar, B. Liu, V.A. Tyurin, V.B. Ritov, A.A. Kapralov, A.A. Amoscato, J. Jiang, T. Anthonymuthu, D. Mohammadyani, Q. Yang, B. Proneth, J. Klein-Seetharaman, S. Watkins, I. Bahar, J. Greenberger, R.K. Mallampalli, B.R. Stockwell, Y.Y. Tyurina, M. Conrad, H. Bayir, Oxidized arachidonic and adrenic PEs navigate cells to ferroptosis, Nat. Chem. Biol. 13 (2017) 81-90.

[43] M. Wortmann, M. Schneider, J. Pircher, J. Hellfritsch, M. Aichler, N. Vegi, P. Kolle, P. Kuhlencordt, A. Walch, U. Pohl, G.W. Bornkamm, M. Conrad, H. Beck, Combined deficiency in glutathione peroxidase 4 and vitamin E causes multiorgan thrombus formation and early death in mice, Circ. Res. 113 (2013) 408-417.
[44] O. Canli, Y.B. Alankus, S. Grootjans, N. Vegi, L. Hultner, P.S. Hoppe, T. Schroeder, P. Vandenabeele, G.W. Bornkamm, F.R. Greten, Glutathione peroxidase 4 prevents necroptosis in mouse erythroid precursors, Blood 127 (2016) 139-148.

[45] S. Hill, C.R. Lamberson, L. Xu, R. To, H.S. Tsui, V.V. Shmanai, A.V. Bekish, A.M. Awad, B.N. Marbois, C.R. Cantor, N.A. Porter, C.F. Clarke, M.S. Shchepinov, Small amounts of isotope-reinforced polyunsaturated fatty acids suppress lipid autoxidation, Free Radic. Biol. Med. 53 (2012) 893-906.

[46] A.Y. Andreyev, H.S. Tsui, G.L. Milne, V.V. Shmanai, A.V. Bekish, M.A. Fomich, M.N. Pham, Y. Nong, A.N. Murphy, C.F. Clarke, M.S. Shchepinov, Isotopereinforced polyunsaturated fatty acids protect mitochondria from oxidative stress, Free Radic. Biol. Med. 82 (2015) 63-72.

[47] J.Y. Cao, S.J. Dixon, Mechanisms of ferroptosis, Cell Mol. Life Sci. 73 (2016) 2195-2209.

\section{Further reading}

[10] M. McDougall, J. Choi, H.-K. Kim, G. Bobe, J.F. Stevens, E. Cadenas, R. Tanguay, M.G. Traber, Lipid quantitation and metabolics outcomes from vitamin E deficient and sufficient zebrafish embyos from 0 to 120h-post-fertillization, Data Brief (2017) (in press). 